\title{
Dynamics of the Rapsyn Scaffolding Protein at the Neuromuscular Junction of Live Mice
}

\author{
Emile G. Bruneau ${ }^{1,2}$ and Mohammed Akaaboune ${ }^{1,2}$ \\ ${ }^{1}$ Department of Molecular, Cellular, and Developmental Biology and ${ }^{2}$ Program in Neuroscience, University of Michigan, Ann Arbor, Michigan 48109
}

The efficacy of synaptic transmission depends on the maintenance of a high density of neurotransmitter receptors and their associated scaffold proteins in the postsynaptic membrane. While the dynamics of receptors has been extensively studied, the dynamics of the intracellular scaffold proteins that make up the postsynaptic density are largely unknown in vivo. Here, we focused on the dynamics of rapsyn, a protein required for the clustering and maintenance of acetylcholine receptor (AChR) density at postsynaptic sites. Using time-lapse imaging, we demonstrated that rapsyn is remarkably dynamic compared to AChRs at functional synapses, turning over 4-6 times more rapidly than AChRs. In addition we found that the rapid turnover of rapsyn is insensitive to alterations in synaptic activity, whereas AChR turnover is profoundly affected, illustrating that rapsyn and receptor dynamics are controlled by distinct mechanisms. These data indicate that individual postsynaptic components are in permanent exchange despite the overall stability of synaptic structure, which may play a role in synaptic plasticity.

\section{Introduction}

The presence of a high density of acetylcholine receptors (AChRs) in the postsynaptic membrane is the hallmark of the neuromuscular junction (NMJ). The accumulation of receptors at synaptic sites is a multistep process controlled by factors ranging from synaptic activity to postsynaptic proteins (Sanes and Lichtman, 2001). In mice lacking the postsynaptic proteins MusK, rapsyn, Dok7, or LRP4, receptor clusters fail to form (Gautam et al., 1995; DeChiara et al., 1996; Okada et al., 2006; Kim et al., 2008; Zhang et al., 2008), and in mice deficient in dystrophin glycoprotein components, the maintenance or stabilization of AChR clusters is compromised (Adams et al., 2000; Grady et al., 2000).

The dynamic movement of receptors between synaptic, nonsynaptic, and internal compartments is the basis of synaptic plasticity. At the mature neuromuscular synapse AChR density is maintained by a dynamic equilibrium of removal, insertion, recycling, and lateral migration mechanisms, all of which appear to be regulated by activity (Akaaboune et al., 1999, 2002; Bruneau et al., 2005). However, the molecular dynamics of individual components that make up the postsynaptic density remain unknown. Also unknown is the extent to which these dynamics may help control synaptic size, density, and stability, and whether this dynamism is modulated by alterations in synaptic activity.

Because rapsyn has been shown to bind directly to AChRs (Burden et al., 1983; LaRochelle and Froehner, 1986) and because of its role in maintaining a normal and stable number and density

Received Sept. 16, 2009; revised Nov. 18, 2009; accepted Nov. 21, 2009.

This work was supported by the National Science Foundation and partially by the National Institutes of Health (M.A.) and National Research Service Award NS056748 (E.G.B.). We thank Daniel Brenner and Isabel Martinez for their technical assistance and the Richard Hume and John Kuwada laboratories for their input and support.

Correspondence should be addressed to Mohammed Akaaboune, Department of Molecular, Cellular, and Developmental Biology, University of Michigan, 830 North University Avenue, Ann Arbor, MI 48109. E-mail: makaabou@umich.edu.

D0I:10.1523/JNEUROSCI.4595-09.2010

Copyright $\odot 2010$ the authors $\quad 0270-6474 / 10 / 300614-06 \$ 15.00 / 0$ of AChRs at the postsynaptic membrane, we sought to investigate how rapsyn is maintained and regulated in vivo. By electroporating rapsyn-GFP into individual muscle fibers and using time-lapse imaging, we show that rapsyn is highly dynamic compared to receptors that occupy the same synapse. Further, in contrast to AChRs, rapsyn insertion appears to be unaffected by alterations in synaptic activity.

\section{Materials and Methods}

Rapsyn construct. The original rapsyn-GFP construct, driven by a CMV promoter, was given as a kind gift by Dr. Jonathan Cohen (Harvard University, Cambridge, MA).

Electroporation. Adult male mice (20-27 g; NSA, Harlan Sprague Dawley) were anesthetized with an intraperitoneal injection of ketamine and xylazine ( 87 and $13 \mathrm{mg}$, respectively, per $\mathrm{kg}$ body weight). The sternomastoid muscle was surgically exposed and $7 \mu \mathrm{l}$ of rapsyn-GFP (2 $\mathrm{mg} / \mathrm{ml}$ ) was layered over the muscle surface. Gold electrodes were placed parallel to the muscle fibers on either side of the muscle, and eight monopolar square-wave pulses were applied perpendicular to the long axis of the muscle. Following electroporation the mouse was sutured and allowed to recover in a heated recovery chamber.

Live animal imaging. Adult male mice were anesthetized with an intraperitoneal injection of ketamine and xylazine, and sternomastoid muscle exposure and NMJ imaging were performed as previously described (Lichtman et al., 1987; Akaaboune et al., 1999). Briefly, the anesthetized mouse was placed on its back on the stage of a customized epifluorescence microscope, and NMJs were imaged (Olympus BW51, Optical Analysis). For imaging at multiple time points, the mouse was sutured and allowed to fully recover between imaging session. All animal usage followed methods approved by the University of Michigan Committee on the Use and Care of Animals.

Quantitative fluorescence imaging. The fluorescence intensity of labeled receptors and rapsyn-GFP at NMJs was assayed using a quantitative fluorescence imaging technique, as described by Turney et al. (1996), with minor modifications.

To determine rapsyn insertion into synaptic regions over time, NMJs on muscles that were electroporated with rapsyn-GFP at least 4 weeks 


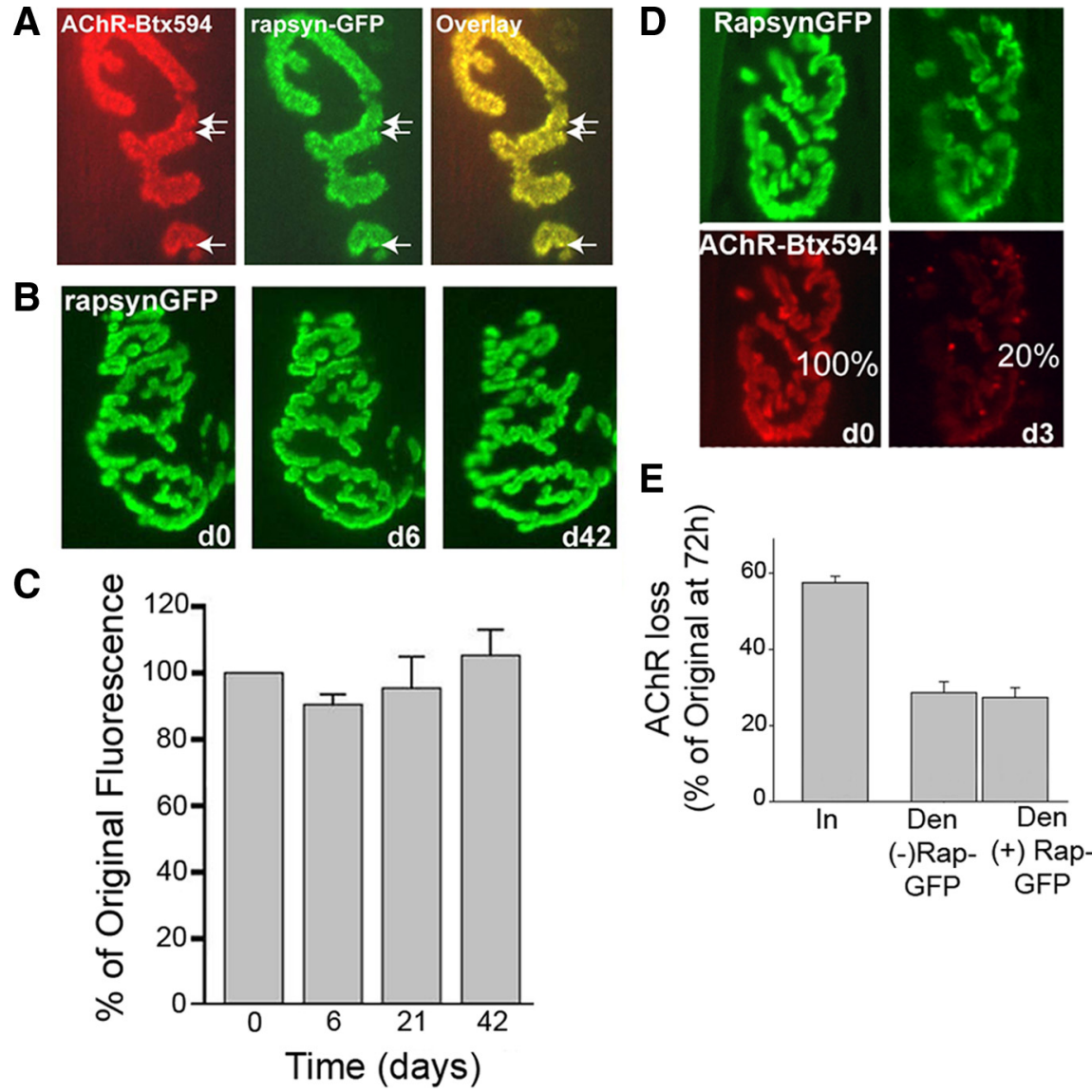

Figure 1. Rapsyn-GFP expression is stable over weeks following electroporation and has no effect on receptor loss. Mouse sternomastoid muscle was electroporated with rapsyn-GFP, and 4 weeks later the muscle was exposed and NMJs were imaged. $A$, A representative synapse showing that rapsyn-GFP fusion protein colocalizes perfectly with AChRs labeled with Btx-594 at synaptic sites. $\boldsymbol{B}$, A representative synapse imaged at multiple time points over 6 weeks shows little change in the overall rapsynGFP density. Images were all taken using the same imaging parameters. C, Quantitative fluorescence data from a number of synapses imaged as in $\boldsymbol{A}$. $\boldsymbol{D}$, Example of a rapsyn-GFP-expressing NMJ on a muscle denervated $8 \mathrm{~d}$ previously that was labeled with a saturating dose of Btx-594 and imaged over $3 \mathrm{~d}$. Note that the presence of rapsyn-GFP does not prevent the accelerated receptor loss at denervated synapses. $\boldsymbol{E}$, Quantification of receptor loss from denervated synapses either devoid of or expressing rapsyn-GFP shows that rapsyn-GFP expression does not slow AChR loss. All data represented as mean \pm SEM. Den, Denervated; In, innervated; Rap-GFP, rapsyn-GFP; d, day.

previously were assayed for GFP expression, and then discrete regions of individual synapses were bleached with an argon laser. The recovery of green fluorescence was quantified both at the bleached region and the unbleached region. Rapsyn-GFP recovery after bleaching was normalized to the nonbleached sections of the same synapse. To determine receptor loss and insertion, two different types of labeling methods were used. For the experiments in the study that involved synapses that were chronically blocked or denervated, Btx-Alexa $594(5 \mu \mathrm{g} / \mathrm{ml}, 1.5 \mathrm{~h})$ was applied to the exposed sternomastoid muscle to saturate all AChRs. Superficial NMJs were then imaged at time 0 and at later time points, and the loss of their fluorescence was assayed. At the end of the experiment, a second dose of the same fluorescent BTX was added to measure the insertion rate of receptors. In another set of experiments, receptors were labeled with either a saturating $(5 \mu \mathrm{g} / \mathrm{ml}, 1.5 \mathrm{~h})$ or a nonsaturating $(2$ $\mu \mathrm{g} / \mathrm{ml}, 2 \mathrm{~min}$ ) dose of Btx-biotin followed by a saturating dose of streptavidin-Alexa $594(10 \mu \mathrm{g} / \mathrm{ml}, 4 \mathrm{~h})$, and their loss of fluorescence over time was assayed. These methods allowed us to estimate AChR removal and insertion and rapsyn insertion at the same synapses over time.

Chronic postsynaptic activity blockade. To investigate rapsyn-GFP and AChR insertion during chronic postsynaptic activity blockade, an exposed sternomastoid muscle that was electroporated with rapsyn-GFP was saturated with Btx-594 and regions of individual synapses were bleached of both GFP and Alexa 594 fluorescence. The muscle was then bathed continuously with D-turbocurare for $8 \mathrm{~h}$ (new curare solution was reapplied every $2 \mathrm{~h}$ for the duration of the experiment). Animals were maintained on a small animal ventilator for the duration of the experiment to prevent asphyxiation. At the end of the experiment, the fluorescent recovery of rapsyn-GFP was measured and compared to the original GFP fluorescence before bleaching. To determine AChR accumulation at the same bleached region over the same time period, Btx-594 ( $5 \mu \mathrm{g} / \mathrm{ml}, 1.5 \mathrm{~h})$ was added to the neck of the mouse before imaging again at $8 \mathrm{~h}$.

Muscle denervation. To determine the effect of denervation on rapsyn-GFP insertion at the postsynaptic membrane, both sternomastoid muscles of adult mice were exposed and electroporated with rapsyn-GFP, and the left sternomastoid muscle was then denervated $21 \mathrm{~d}$ later and prevented from reinnervating by excising a $5 \mathrm{~mm}$ piece of the sternomastoid nerve.

\section{Results}

To study the molecular dynamics of rapsyn at individual synapses in vivo, we first asked whether rapsyn is expressed and stable over time. For this, the sternomastoid muscle of live mice was electroporated with plasmid containing a recombinant rapsyn-GFP construct. We found that the rapsyn-GFP fusion protein colocalized perfectly with AChRs at synaptic sites (Fig. 1A). To be sure that overall rapsyn-GFP expression did not change over the time period of our experiments (hours to days), we monitored changes in the fluorescence of rapsyn-GFP at individual synapses over time in mice that had been electroporated 4 or 6 weeks previously by assaying the GFP fluorescence at individual synapses expressing rapsynGFP over time. The fluorescence intensity at each time point was quantified (Fig. $1 B)$. We found that the average rapsynGFP expression was $87 \pm 16 \%$ (SD, $n=31$ ) of original fluorescence after $3 \mathrm{~d}, 91 \pm 16 \%(\mathrm{SD}, n=27)$ after $6 \mathrm{~d}, 95 \pm 25 \%(\mathrm{SD}$, $n=14$ ) after $21 \mathrm{~d}$, and $105 \pm 27 \%(\mathrm{SD}, n=12)$ after $42 \mathrm{~d}$ (Fig. $1 B, C)$. This indicates that rapsyn-GFP expression is relatively stable over long periods of time.

It has been shown previously that increased expression of rapsyn at synapses slows AChR turnover. If our rapsyn-GFP construct added to the total rapsyn density at synapses, we would predict an increase in AChR lifetime at the synapse. Conversely, if the rapsyn-GFP construct replaced endogenous rapsyn to keep rapsyn density constant, we would expect there to be no change in AChR turnover at synapses expressing rapsyn-GFP. To examine this, muscles that had previously been electroporated with rapsyn-GFP were sequentially labeled with saturating doses of Btx-biotin and streptavidin-Alexa 594 to label all AChRs. We then monitored the loss of labeled AChRs from superficial synapses on the same muscle from synapses that were both expressing rapsyn-GFP and not expressing rapsyn-GFP. Three days after the initial labeling, $58 \pm 5 \%(\mathrm{SD}, n=15)$ of the original AChR fluorescent signal remained at synapses devoid of rapsyn-GFP, and $60 \pm 6 \%(\mathrm{SD}, n=14)$ remained at synapses that were ex- 
pressing some visible level of rapsyn-GFP. This indicates that the loss of receptors was independent of rapsyn-GFP expression in synapses. We further explored whether the presence of rapsynGFP would prevent accelerated AChR loss observed at NMJs on denervated muscles. To do this the left sternomastoid muscle expressing rapsyn-GFP that had been surgically denervated $8 \mathrm{~d}$ previously was exposed, labeled with Btx-594, and imaged. Three days later, we found $28 \pm 5 \%$ (SD, $n=17$ ) of fluorescence remained at denervated synapses expressing rapsyn-GFP and $27 \pm$ $4 \%(\mathrm{SD}, n=11)$ remained at denervated synapses without rapsyn-GFP, while $57 \pm 4 \%(\mathrm{SD}, n=21)$ remained at synapses on the non-denervated muscle (Fig. $1 D, E$ ). These data indicate that rapsyn-GFP expression at synapses does not slow the accelerated AChR removal. Finally, immunohistochemistry was performed on muscle fibers expressing different levels of rapsynGFP using an anti-rapsyn antibody that has been shown previously to recognize both rapsyn and rapsyn-GFP fusion proteins (Gervásio and Phillips, 2005). Using quantitative immunofluorescence, we found that the overall density of rapsyn at synapses expressing rapsyn-GFP (13.46 $\pm 4 \mathrm{SD}$, arbitrary units, $n=15)$ was not significantly different from rapsyn density at NMJs not expressing rapsyn-GFP $(12.85 \pm 3.05 \mathrm{SD}$, arbitrary units, $n=14)$ (supplemental Fig. 1, available at www.jneurosci.org as supplemental material).

Having found that rapsyn-GFP colocalizes perfectly with AChRs at synapses, is able to cluster AChRs similarly to endogenous rapsyn, does not alter the overall rapsyn density or normal AChR dynamics, and is synaptically expressed at a constant level over the time window of our experiments, we felt comfortable using this construct to estimate the rate of rapsyn turnover at synapses in vivo. Adult mice were electroporated with rapsynGFP, and $\sim 4$ weeks after electroporation the sternomastoid muscle was reexposed and NMJs expressing rapsyn-GFP were imaged. Discrete portions of individual junctions were carefully traced with an argon laser to remove the fluorescence without altering fluorescent expression at other regions of the NMJ. When the recovery of fluorescence at the bleached portion of each junction was measured at later time points and normalized to the amount of rapsyn-GFP expression at unbleached region of the same junction, we found that $61 \pm 17 \%(\mathrm{SD}, n=32)$ and $83 \pm 14 \%(\mathrm{SD}, n=14)$ of fluorescence was recovered at bleached regions after 24 , and $72 \mathrm{~h}$, respectively (Figs. $2 A, B$ ). The recovery of fluorescence at bleached areas was similar in synapses expressing low or high levels of rapsyn-GFP (Fig. 2C), suggesting the rapid recovery is not dependent on the expression level of exogenous rapsyn.

Next we asked whether receptors and rapsyn have similar synaptic lifetimes. To measure AChR and rapsyn-GFP dynamics at the same synapse, we bleached rapsyn-GFP, and then immediately labeled the AChRs with a fluorescent tag. In this way we could measure the recovery of rapsyn-GFP fluorescence and the loss of AChR-Alexa fluorescence (AChR removal) simultaneously. To do this, the sternomastoid muscles of mice that were electroporated with rapsyn-GFP were labeled with a subsaturating dose of Btx-biotin (to label a small proportion of the receptors $(<20 \%)$ and thus allow the synapses to remain active), discrete regions of synapses expressing rapsyn-GFP were bleached, and the synapse was imaged. The sternomastoid muscle was then immediately incubated with streptavidin-Alexa 594 (strept-594) to label all biotin sites and NMJs were imaged. We found that only $5 \mathrm{~h}$ after rapsyn-GFP bleaching, $26 \pm 10 \%(\mathrm{SD}, n=14)$ of original GFP fluorescence had recovered at bleached sites. After $29 \mathrm{~h}$ rapsyn-GFP insertion was $62 \pm 19 \%(\mathrm{SD}, n=18)$ of original
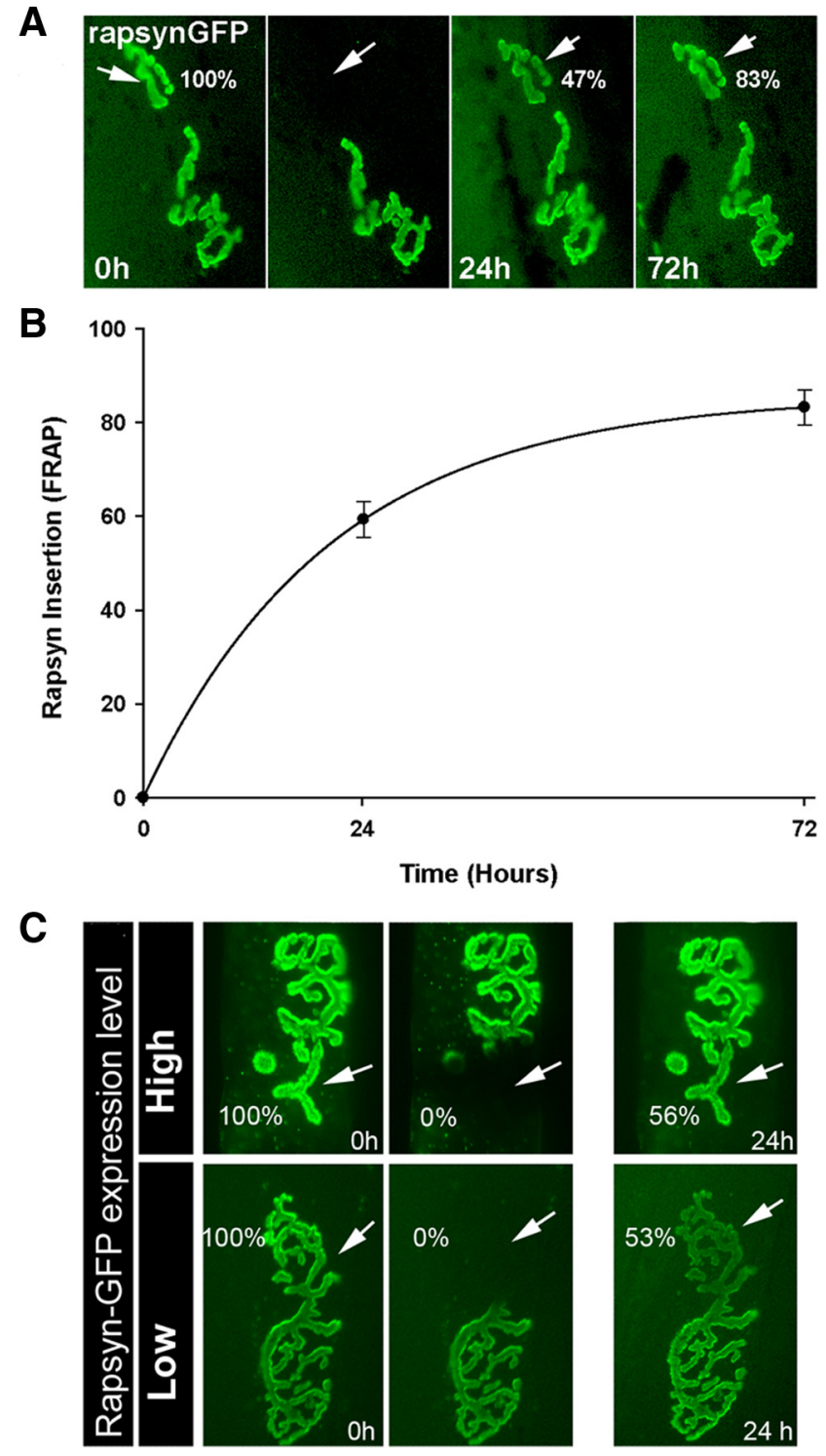

Figure 2. Rapsyn-GFP fluorescence is recovered rapidly at bleached synapses. Sternomastoid muscles of living mice were electroporated with rapsyn-GFP, and 4 weeks later the muscles were reexposed, and synapses expressing rapsyn-GFP were imaged. The fluorescence from discrete sections of individual junctions was then removed with a laser, and the recovery of fluorescence was monitored over $3 \mathrm{~d}$. $\boldsymbol{A}$, Example of a neuromuscular junction that was imaged at time 0 and immediately bleached and reimaged at 24 and 72 h. $B$, Graph summarizing data obtained from many synapses. All data represent mean \pm SEM. $C$, Representative images showing that the recovery of rapsyn-GFP is nearly equal at synapses expressing low or high rapsyn-GFP level.

fluorescence, and after $77 \mathrm{~h}$ insertion was $89 \pm 12 \%(\mathrm{SD}, n=16)$ of original fluorescence. This rate of insertion was similar to the insertion after 24 and $72 \mathrm{~h}$ when rapsyn-GFP-expressing synapses were bleached in the absence of any bungarotoxin (Fig. 2). Receptor removal at the same synapses over the same 24 and $72 \mathrm{~h}$ time periods were $7 \pm 5 \%(\mathrm{SD}, n=5)$ and $25 \pm 8 \%(\mathrm{SD}, n=5)$ of original fluorescence, respectively (Fig. $3 A, C$ ). These data indicate that rapsyn is much more dynamic than AChRs.

Similar experiments were also performed on junctions that had been initially labeled with saturating doses of Btx-biotin and strept-594, which transiently blocks muscle action potentials until the progressive insertion of new, unlabeled receptors over time restore activity. After 5, 29, and $77 \mathrm{~h}$, rapsyn-GFP insertion (after 
A

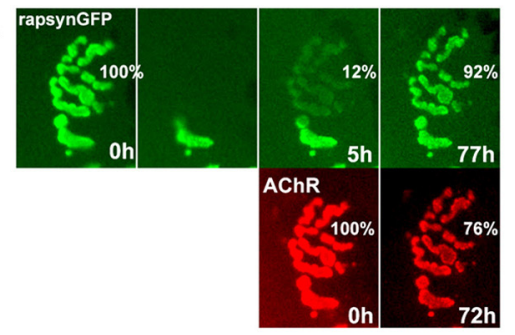

B

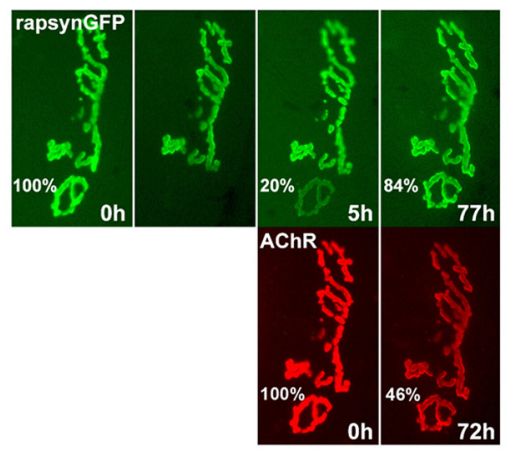

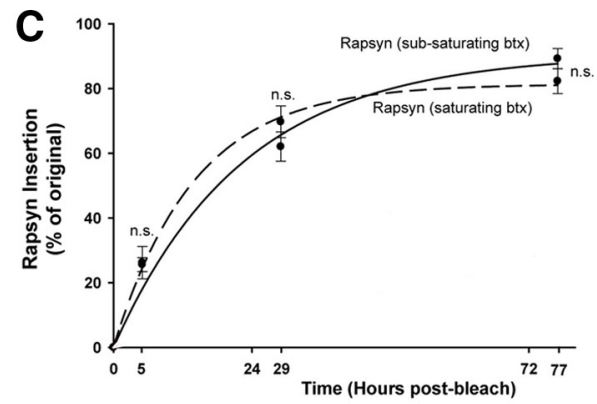

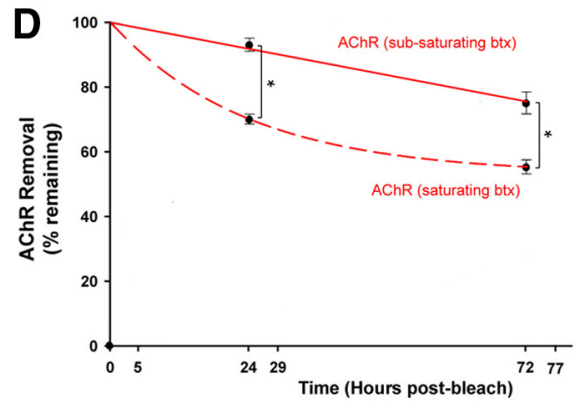

Figure 3. Rapsyn and AChR dynamics at the NMJ. The sternomastoid muscle was exposed, rapsyn-GFP was bleached, and muscles were immediately incubated in either saturating $(\boldsymbol{A})$ or nonsaturating $(\boldsymbol{B})$ doses of Btx-biotin followed by a saturating dose of strept-594. After labeling, synapses were imaged, and initial fluorescence from AChRs and recovered fluorescence from rapsynGFP was measured. After 1 and/or 3 d, the muscle was reexposed, and the same synapses were again imaged. C, D, Receptor loss (D) and rapsyn insertion ( $\boldsymbol{C}$ from many synapses measured as in $\boldsymbol{A}$ and $\boldsymbol{B}$. Note that $\mathrm{AChR}$ removal is significantly and dramatically altered at saturated (postsynaptic activity blocked) versus unsaturated (postsynaptic activity not blocked) synapses $\left({ }^{*} p<0.05\right.$ ), while rapsyn insertion at these same synapses is unaffected by activity blockade ( $p>0.05$ at all time points, n.s.). All data represent mean \pm SEM.

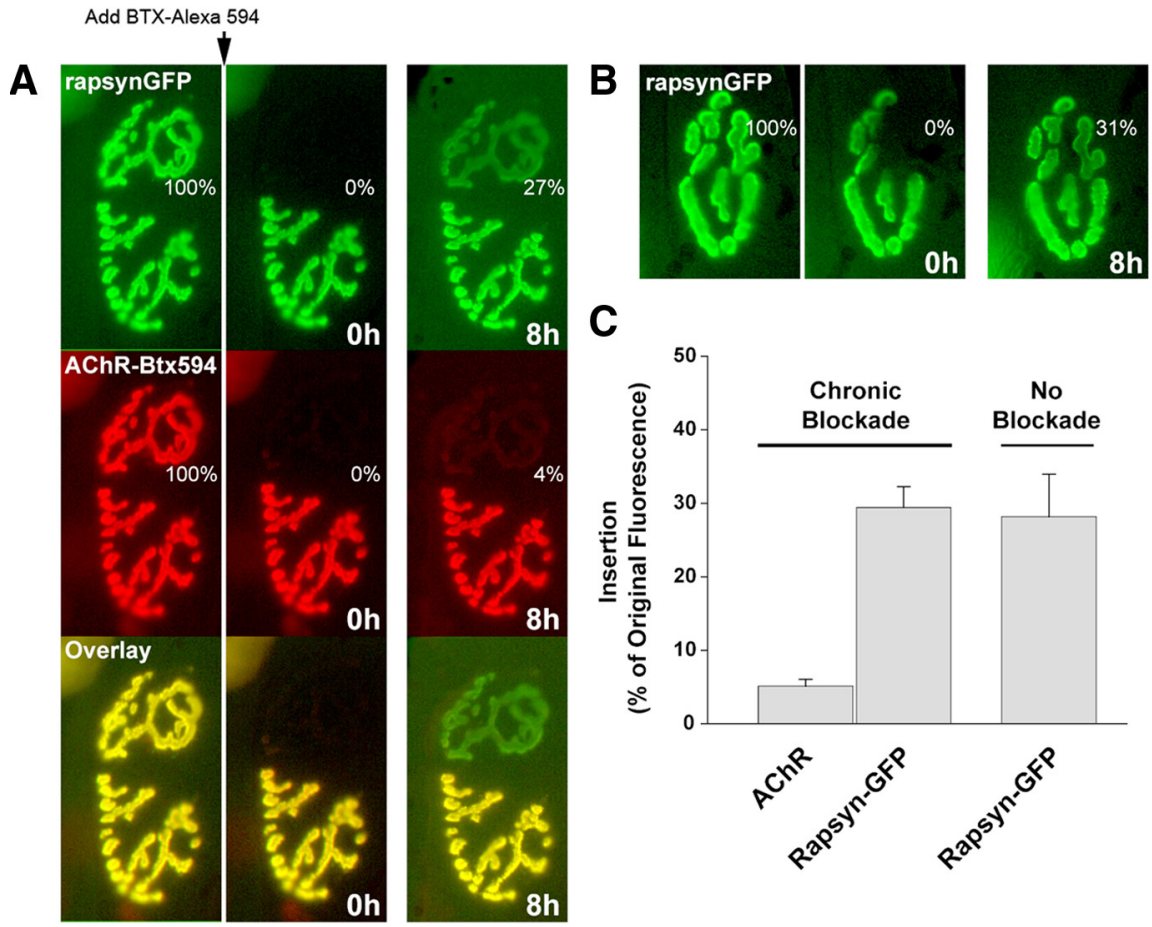

Figure 4. Rapsyn-GFP synaptic turnover is not altered by synaptic activity blockade. Sternomastoid muscles of live mice electroporated with rapsyn-GFP were bathed with a saturating dose of Btx-594. $A$, The doubly labeled NMJs (expressing rapsyn-GFP and fluorescent Btx) were imaged, the GFP fluorescence from rapsyn-GFP and the Alexa 594 fluorescence from AChR-Btx- 594 were removed from discrete regions of synapses with a laser, and the muscle was immediately incubated in the AChR antagonist, curare, for the duration of the experiment. After $8 \mathrm{~h}$, fresh Btx-594 was applied to label any newly inserted receptors, and images were again obtained. Note that rapsyn insertion is far greater than AChR insertion at the same synapse. $\boldsymbol{B}$, A sample image of a synapse that was imaged and bleached of rapsyn-GFP fluorescence in the absence of any postsynaptic activity blockade. $C$, Data obtained from 7 and 13 synapses from experiments done as in $\boldsymbol{A}$ and $\boldsymbol{B}$, respectively. Data represent mean \pm SEM.

photobleaching) was $26 \pm 12 \%$ (SD, $n=$ $34), 70 \pm 20 \%$ (SD, $n=26)$, and $82 \pm 16 \%$ (SD, $n=17$ ) respectively (Fig. $3 B, C$ ). At the same synapse, receptor removal was $30 \pm 10 \%(\mathrm{SD}, n=40)$ and $45 \pm 7 \%(n=$ 11) of original fluorescence at 24 and $72 \mathrm{~h}$ (Fig. 3B). These data indicate that rapsyn insertion may be unaffected by transient AChR blockade and therefore independent of postsynaptic activity. This led us to directly examine the effect of synaptic activity on rapsyn insertion.

Previous studies have shown that the insertion of receptors into the postsynaptic membrane is regulated by synaptic activity. Since rapsyn binds directly to receptors, we asked whether postsynaptic rapsyn dynamics are also manipulated by synaptic activity. To investigate this, sternomastoid muscles electroporated with rapsyn-GFP were labeled with bungarotoxin-Alexa 594 (Btx-594), and then both the GFP and Alexa 594 fluorescence were removed from discrete regions of individual NMJs with an argon laser. The sternomastoid muscles were then continuously bathed with the AChR antagonist curare to chronically block muscle activity for $8 \mathrm{~h}$. At the end of the experiment, the sternomastoid muscle was again bathed with Btx-594 to label new receptors that had been inserted into the synapse over this time. We found that AChR insertion was only $5 \pm 2 \%$ (SD, $n=$ 7) of original fluorescence, while rapsynGFP recovery was $29 \pm 8 \%$ SD of original fluorescence. When we monitored the recovery of rapsyn-GFP at synapses that had been only transiently blocked with bungarotoxin (an initial, saturating dose of Btx$594)$ or that were without blockade, we found that the rapsyn-GFP fluorescent recovery rates were similar to recovery at chronically blocked synapses (Fig. $4 B, C$ ). The change in fluorescence at unbleached portions of chronically blocked synapses was negligible (an increase of $4 \pm 5 \%$ SD of original fluorescence), indicating that synaptic activity has no effect on either the loss or insertion of rapsyn-GFP at synaptic sites. This is in stark contrast to the dramatic effect that activity blockade has on AChR dynamics.

\section{Discussion}

In these studies we took advantage of a green fluorescent fusion protein to investigate the dynamics of the inaccessible intracellular scaffold protein, rapsyn, at the neuromuscular junction of living mice. We found that the turnover rate of rapsyn sharply contrasts with receptors at the same postsynaptic density of functioning synapses: rapsyn turns over at synapses 
$4-6$ times more rapidly $\left(t_{1 / 2}\right.$ of $\left.\sim 1 \mathrm{~d}\right)$ than receptors $\left(t_{1 / 2} \sim 6 \mathrm{~d}\right)$. In addition, we found that alterations in synaptic activity had distinct effects on the dynamics of rapsyn and receptors at the same synapse: when postsynaptic activity was blocked, receptor lifetime was dramatically reduced while rapsyn lifetime remained unchanged. Therefore, despite the intimate association of rapsyn with receptors, our data indicate that receptors and rapsyn are regulated independently at the same synapses.

In contrast to previous studies showing that the overexpression of rapsyn through electroporation of rapsyn-GFP into muscle cells in vivo can stabilize receptors (Gervásio and Phillips, 2005), our results indicate that rapsyn-GFP expression at clusters did not alter AChR stability. Since overall rapsyn density was similar at synapses expressing rapsyn-GFP and at synapses devoid of the fusion protein, it seems likely that exogenous rapsyn-GFP is able to replace endogenous rapsyn at clusters without increasing rapsyn density; alternatively the number of exogenous rapsyn-GFP molecules inserted at clusters was negligible despite the strong GFP fluorescence signal. Regardless, in our study rapsyn-GFP functioned like endogenous rapsyn (it specifically clustered and colocalized with receptors at NMJs, its expression did not alter the clustering, number, or density of receptors at the $\mathrm{NMJ}$ ), and did not alter receptor stability on either innervated or denervated muscles.

The most significant result of the current study is the highly transient nature of rapsyn in the postsynaptic density. It is possible that this behavior may be explained by a permanent exchange between bound and unbound forms of rapsyn. This raises the question of what role rapid rapsyn dynamics may have in regulating postsynaptic AChR dynamics and overall synaptic AChR density over time. It is conceivable that the high dynamism of rapsyn may have an impact on the rates of insertion, removal, or recycling of receptors at the NMJ, which serve as the basis for synaptic plasticity. Indeed the importance of rapsyn in the regulation of receptor density at synaptic sites has been shown in myasthenia syndrome, in which the receptor number is significantly decreased, and consequently the safety factor of the NMJ is compromised.

Although the present study represents the first time that intracellular protein dynamics have been investigated at the NMJ in vivo, a small number of other studies have examined the dynamics of scaffolding proteins at other synapses. These studies have found that postsynaptic scaffold proteins at excitatory and inhibitory synapses exhibit similar fast dynamics relative to the receptors anchored at the postsynaptic site. For example, studies of PSD-95, which binds directly to NMDA receptors and indirectly to AMPA receptors and nicotinic AChRs in the CNS, have found that the exchange rate of individual PSD-95 molecules is much faster than the associated receptors, as determined by FRAP(Okabe et al., 2001; Rasse et al., 2005; Gray et al., 2006). At glycinergic synapses, the glycine receptor-associated protein gephyrin has also been shown to turn over rapidly (Hanus et al., 2006). Interestingly, similar to rapsyn, gephyrin turnover in this study was also shown to be regulated differently from the receptors to which they bind.

Finally, our work raises the question of why AChR dynamics are dramatically altered by activity while rapsyn-GFP are unaffected. One potential explanation for these data is that rapsyn insertion may be insensitive to changes in postsynaptic activity, but regulated instead by nerve factors. Consistent with this, agrin signaling has been shown to increase the expression and targeting of rapsyn to the postsynaptic membrane (Brockhausen et al.,
2008). It would also be worthwhile to see whether rapsyn is subject to palmitoylation and depalmitoylation or ubiquitination processes. Such mechanisms have been reported for scaffold proteins at the excitatory synapses (Topinka and Bredt, 1998; El-Husseini and Bredt, 2002), which could be involved in synaptic remodeling and plasticity.

The results reported here suggest that, despite the stability of the synaptic structure over the lifetime of the animal (BaliceGordon and Lichtman, 1990), individual molecules such as rapsyn and receptors are highly dynamic and are constantly exchanged between the postsynaptic membrane and internal compartments. Extending this investigation to other scaffolding proteins of the synapse might allow better understanding of the dynamic processes behind plasticity.

\section{References}

Adams ME, Kramarcy N, Krall SP, Rossi SG, Rotundo RL, Sealock R, Froehner SC (2000) Absence of alpha-syntrophin leads to structurally aberrant neuromuscular synapses deficient in utrophin. J Cell Biol 150:13851398.

Akaaboune M, Culican SM, Turney SG, Lichtman JW (1999) Rapid and reversible effects of activity on acetylcholine receptor density at the neuromuscular junction in vivo. Science 286:503-507.

Akaaboune M, Grady RM, Turney S, Sanes JR, Lichtman JW (2002) Neurotransmitter receptor dynamics studied in vivo by reversible photounbinding of fluorescent ligands. Neuron 34:865-876.

Balice-Gordon RJ, Lichtman JW (1990) In vivo visualization of the growth of pre- and postsynaptic elements of neuromuscular junctions in the mouse. J Neurosci 10:894-908.

Brockhausen J, Cole RN, Gervásio OL, Ngo ST, Noakes PG, Phillips WD (2008) Neural agrin increases postsynaptic ACh receptor packing by elevating rapsyn protein at the mouse neuromuscular synapse. Dev Neurobiol 68:1153-1169.

Bruneau E, Sutter D, Hume RI, Akaaboune M (2005) Identification of nicotinic acetylcholine receptor recycling and its role in maintaining receptor density at the neuromuscular junction in vivo. J Neurosci 25:9949-9959.

Burden SJ, DePalma RL, Gottesman GS (1983) Crosslinking of proteins in acetylcholine receptor-rich membranes: association between the betasubunit and the $43 \mathrm{kd}$ subsynaptic protein. Cell 35:687-692.

DeChiara TM, Bowen DC, Valenzuela DM, Simmons MV, Poueymirou WT, Thomas S, Kinetz E, Compton DL, Rojas E, Park JS, Smith C, DiStefano PS, Glass DJ, Burden SJ, Yancopoulos GD (1996) The receptor tyrosine kinase MuSK is required for neuromuscular junction formation in vivo. Cell 85:501-512.

El-Husseini AE, Bredt DS (2002) Protein palmitoylation: a regulator of neuronal development and function. Nat Rev Neurosci 3:791-802.

Gautam M, Noakes PG, Mudd J, Nichol M, Chu GC, Sanes JR, Merlie JP (1995) Failure of postsynaptic specialization to develop at neuromuscular junctions of rapsyn-deficient mice. Nature 377:232-236.

Gervásio OL, Phillips WD (2005) Increased ratio of rapsyn to ACh receptor stabilizes postsynaptic receptors at the mouse neuromuscular synapse. J Physiol 562:673-685.

Grady RM, Zhou H, Cunningham JM, Henry MD, Campbell KP, Sanes JR (2000) Maturation and maintenance of the neuromuscular synapse: genetic evidence for roles of the dystrophin-glycoprotein complex. Neuron 25:279-293.

Gray NW, Weimer RM, Bureau I, Svoboda K (2006) Rapid redistribution of synaptic PSD-95 in the neocortex in vivo. PLoS Biol 4:e370.

Hanus C, Ehrensperger MV, Triller A (2006) Activity-dependent movements of postsynaptic scaffolds at inhibitory synapses. J Neurosci 26:4586-4595.

Kim N, Stiegler AL, Cameron TO, Hallock PT, Gomez AM, Huang JH, Hubbard SR, Dustin ML, Burden SJ (2008) Lrp4 is a receptor for Agrin and forms a complex with MuSK. Cell 135:334-342.

LaRochelle WJ, Froehner SC (1986) Determination of the tissue distributions and relative concentrations of the postsynaptic $43-\mathrm{kDa}$ protein and the acetylcholine receptor in Torpedo. J Biol Chem 261:5270-5274.

Lichtman JW, Magrassi L, Purves D (1987) Visualization of neuromuscular 
junctions over periods of several months in living mice. J Neurosci 7:1215-1222.

Okabe S, Urushido T, Konno D, Okado H, Sobue K (2001) Rapid redistribution of the postsynaptic density protein PSD-Zip45 (Homer 1c) and its differential regulation by NMDA receptors and calcium channels. J Neurosci 21:9561-9571.

Okada K, Inoue A, Okada M, Murata Y, Kakuta S, Jigami T, Kubo S, Shiraishi H, Eguchi K, Motomura M, Akiyama T, Iwakura Y, Higuchi O, Yamanashi Y (2006) The muscle protein Dok-7 is essential for neuromuscular synaptogenesis. Science 312:1802-1805.

Rasse TM, Fouquet W, Schmid A, Kittel RJ, Mertel S, Sigrist CB, Schmidt M, Guzman A, Merino C, Qin G, Quentin C, Madeo FF, Heckmann M,
Sigrist SJ (2005) Glutamate receptor dynamics organizing synapse formation in vivo. Nat Neurosci 8:898-905.

Sanes JR, Lichtman JW (2001) Induction, assembly, maturation and maintenance of a postsynaptic apparatus. Nat Rev Neurosci 2:791-805.

Topinka JR, Bredt DS (1998) N-terminal palmitoylation of PSD-95 regulates association with cell membranes and interaction with $\mathrm{K}+$ channel Kv1.4. Neuron 20:125-134.

Turney SG, Culican SM, Lichtman JW (1996) A quantitative fluorescenceimaging technique for studying acetylcholine receptor turnover at neuromuscular junctions in living animals. J Neurosci Methods 64:199-208.

Zhang B, Luo S, Wang Q, Suzuki T, Xiong WC, Mei L (2008) LRP4 serves as a coreceptor of agrin. Neuron 60:285-297. 